\title{
Restauración adaptable en la Meseta PurépeCha, MICHOACÁN, MÉXICO: HACIA UN MODELO DE ESTADOS Y TRANSICIONES
}

\author{
Roberto Lindig-Cisneros ${ }^{1,4}$, Arnulfo Blanco-García', Cuauhtémoc Sáenz-Romero², \\ Pedro Alvarado-Sosa ${ }^{3}$ y Nancy Alejandre-Melena ${ }^{3}$
}

\begin{abstract}
${ }^{1}$ Centro de Investigaciones en Ecosistemas, Universidad Nacional Autónoma de México, Antigua Carr. a Pátzcuaro No. 8701, Col. Ex Hacienda de San José de la Huerta, C.P. 58190, Morelia, Michoacán, México. ${ }^{2}$ Instituto de Investigaciones Sobre los Recursos Bióticos, Universidad Michoacana de San Nicolás de Hidalgo, Apdo. Postal 18, Administración 3 Santa María, C.P. 58091, Morelia, Michoacán, México.

${ }^{3}$ Laboratorio de Ecología de Restauración, Facultad de Biología, Universidad Michoacana de San Nicolás de Hidalgo, Apdo. Postal 18, Administración 3 Santa María, C.P. 58091, Morelia, Michoacán, México.

${ }^{4}$ Autor para la correspondencia. Correo-e: rlindig@oikos.unam.mx
\end{abstract}

\begin{abstract}
Resumen: La restauración ecológica requiere de técnicas apropiadas que dirijan al sistema hacia las metas deseadas. La combinación de factores de disturbio antrópicos y naturales pueden crear estados en los cuales la sucesión secundaria no lleva al sistema hacia estadios maduros de la vegetación o lo hace de manera muy lenta. En el presente trabajo se desarrolla un esquema conceptual siguiendo los modelos de estados y transiciones para la restauración de bosques de pino en la Meseta Purépecha. Se sintetiza la información obtenida de un proyecto de restauración adaptable que se inició de manera colaborativa con la comunidad indígena de Nuevo San Juan Parangaricutiro en 2001. Se describe un estado posiblemente estable, los depósitos de ceniza volcánica conocidos como "arenales", y se detallan algunas técnicas de restauración que permitirían su transición hacia el estado deseable dominado por Pinus pseudostrobus.
\end{abstract}

Palabras clave: acolchado, pinos, restauración, supervivencia.

\begin{abstract}
Ecological restoration requires appropriate techniques for reaching desired goals. The combination of human and natural disturbances can create states that prevent the development of mature forests through secondary succession, or can slow down the transition. A conceptual scheme based on state and transition models is developed for pine forest restoration in the Purépecha Plateau. Information gathered from an adaptive restoration project initiated in 2001 in collaboration with the Indigenous Community of Nuevo San Juan Parangaricutiro is synthesized. One stable state is described, namely tephra deposits locally known as arenales, and restoration techniques for making the transition to a desirable state dominated by Pinus pseudostrobus are examined.
\end{abstract}

Key words: mulch, pines, restoration, survival

$\mathrm{C}$ omo en muchas ocasiones no se cuenta con el conocimiento necesario al iniciar un proyecto de restauración ecológica, se requiere del desarrollo de técnicas y estrategias de manejo apropiadas que permitan la generación del conocimiento necesario y a la vez dirigir al sistema de interés hacia las metas esperadas. Se han utilizado varios modelos para organizar la investigación y la implementación de las acciones de restauración ecológica. Entre ellos, destaca el modelo de Bradshaw (1984), el cual consiste en llevar un sistema degradado hasta una meta consistente en restablecer el sistema que existía antes de la perturbación o uno comparable a un sistema no perturbado, que es usado como ecosistema de referencia. Cuando no se llega a ninguna de estas metas se considera como una recuperación o un reemplazo (Bradshaw, 1984). Dos aspectos fundamentales del modelo de Bradshaw es que considera 
que la restauración sigue el mismo camino que la sucesión (primaria o secundaria) y que supone una relación muy simple entre el proceso de restauración y la recuperación de atributos estructurales y funcionales. Otros modelos han considerado el efecto del nivel de disturbio sobre la naturaleza e intensidad del esfuerzo requerido para lograr la restauración (Hobbs y Norton, 1996), el papel de los alrededores (comunidades circundantes, ecosistemas o el paisaje) sobre los resultados en el sitio de restauración (Palmer et al., 1997) o el efecto del tiempo en los resultados de la restauración (Hobbs y Mooney, 1993).

A pesar del desarrollo de los modelos anteriores y otros, predecir los resultados de un esfuerzo de restauración en un sitio en particular es aún difícil (Zedler y Callaway, 2000). Esto se puede atribuir a dos causas principales: (1) a que las condiciones que se encuentran en los sitios de restauración rara vez coinciden con las condiciones en que actúan los procesos sucesionales (Zedler, 2000) y por lo tanto la dinámica de muchos procesos ecosistémicos en sitios sujetos a restauración ecológica es desconocida; y (2) a que las condiciones de muchos sitios son tan severas, que llevar al sistema a condiciones similares a las históricas o las de sistemas naturales de referencia existentes es imposible (Lindig-Cisneros y Zedler, 2000).

Los modelos de estados y transiciones (state and transi tion models) que fueron propuestos originalmente para el manejo de zonas de pastoreo (Westoby et al., 1989), han sido incorporados a la restauración ecológica (Hobbs y Norton, 1996; Yates y Hobbs, 1997), pues permiten conceptualizar las barreras que se deben superar para lograr las metas de restauración deseadas. Los modelos de estados y transiciones surgieron por la incapacidad de los modelos clásicos, basados en la teoría sucesional gradualista, de explicar los cambios en tierras de agostadero causados por diversas prácticas de manejo que causan, entre otros efectos negativos, la dominancia de especies arbustivas e invasoras (Briske et al., 2005).

Los modelos de estados y transiciones se desarrollaron para comprender la dinámica de la vegetación en la escala temporal que es relevante para el manejo, que en muchos tipos de ecosistemas puede ser menor a la de los procesos sucesionales. Estos modelos reconocen que existen múltiples estados, alternativos, casi estables o estables, de acuerdo con el concepto de estados estables múltiples (Holling, 1973; May, 1977) que se mantiene debido a las interacciones bióticas y los regímenes de disturbio, siguiendo el paradigma del no-equilibrio (Ellis y Swift, 1988).

Un estado se define como una comunidad vegetal persistente que no es fácilmente alterable a través de un proceso lineal de cambio (Tamzen et al., 2001). Los estados se han definido de manera descriptiva (Westoby et al., 1989) o estadística (Allen-Diaz y Bartolome, 1998). Cuando se alteran los regímenes de disturbio, por ejemplo por medio de incendios intencionales, se puede transitar a estados altemativos. Estas transiciones entre estados pueden ocurrir de manera rápida o lenta, pero la composición de la comunidad no deja de cambiar a lo largo de la transición. Cuando se habla de estados alternativos, no es una condición indispensable que los estados sean estables a largo plazo, pues basta con que la composición y la estructura se mantengan dentro de un intervalo tal que no se pueda transitar (es decir, que existan distribuciones multimodales de composición) en el lapso de tiempo que es relevante para el manejo. Para que un estado se pueda considera estable en un sentido más estricto, además de la condición de multimodalidad, se requieren tres condiciones más: (1) que existan procesos de retroalimentación positiva que mantengan el estado (por ejemplo el fuego que favorece a los pastos que a su vez son favorecidos por el fuego); (2) que la transición entre estados sea no lineal, es decir, que haya un "brinco temporal" en las variables que describen al sistema, y finalmente, (3) que haya histéresis en el proceso, es decir, que el camino que condujo al cambio entre estados sea diferente del que conduce al cambio en sentido inverso (Van Nes et al., 2002; Suding et al., 2004; Briske et al., 2005).

Algunos de los estados alternativos que se generan por las actividades humanas pueden ser estables en un sentido estricto, es decir, se cumplen las condiciones antes descritas. Éste sería el caso de la sucesión detenida. También puede ocurrir que a partir de estos estados alternativos la sucesión siga caminos diferentes de los que seguiría bajo condiciones normales (entendidas éstas como las condiciones que existían antes de la intervención humana).

Un aspecto de gran relevancia para la restauración ecológica es que los modelos de estados y transiciones implican que se deben dar dinámicas no lineales o transiciones (umbrales sensu Hobbs y Norton, 1996), que dificulten o impidan que se retorne a estados sucesionales avanzados o deseables (ya sea estructural o funcionalmente). A su vez, esto implica que algunos estados pueden ser irreversibles (Zedler, 2000; Lindig-Cisneros et al., 2003).

Debido a las variaciones tanto en número como en intensidad de las medidas necesarias para transitar de estados degradados hacia estados estables (Zedler, 1999), el manejo adaptable de la restauración se vuelve la estrategia más eficaz para la implementación de la restauración ecológica. La "restauración adaptable" es un esquema de manejo adaptable aplicado a la restauración que consiste en la instrumentación de una serie de medidas alternativas, la evaluación de sus resultados y la integración del conocimiento adquirido a etapas subsecuentes del manejo del ecosistema para dirigirlo hacia las metas deseadas (Christensen et al., 1996; Zedler, 2003).

El presente trabajo tiene como objetivo presentar un esquema conceptual basado en los modelos de estados y transiciones para la restauración de bosques de coníferas en 
la Meseta Purépecha. Se describe un estado alternativo (posiblemente estable), los depósitos de ceniza volcánica conocidos como "arenales", y se detallan algunas técnicas de restauración que permitirían su transición hacia el estado deseable dominado por Pinus pseudostrobus.

\section{El proyecto de restauración adaptable de bosques de coníferas en la Meseta Purépecha}

En septiembre de 2001 se comenzó un proyecto de restauración adaptable en colaboración con la Dirección Técnica Forestal de la comunidad indígena de Nuevo San Juan Parangaricutiro (CINSJP), Michoacán, con la finalidad de desarrollar técnicas de restauración adecuadas para ser incorporadas a esquemas de manejo sustentable de los recursos forestales. Desde junio de 2002, cuando se inició el trabajo experimental, hasta la fecha se han concluido tres etapas del proyecto y una cuarta está en proceso. En el presente trabajo sintetizamos la información generada en las tres primeras etapas que están concluidas y cuyos resultados han sido publicados, ya sea en revistas especializadas o en forma de tesis.

La primera etapa consistió en el estudio de la vegetación natural presente en los arenales de origen volcánico (Lindig-Cisners et al., 2006), y de las plantaciones de pinos establecidas en los arenales (Alejandre-Melena, 2004). Esta etapa se complementó con estudios controlados para determinar el efecto de la profundidad de la capa de ceniza volcánica en el desempeño de diversas especies (Gómez-Romeroet al., 2006). La segunda etapa consistió en un ex perimento de restauración para poner a pru eba el e fecto del acolchado en la supervivencia de Pinus pseu dostrobus (de entre 7 y 8 meses de edad al momento de ser plantados) y Lupinus elegans (Blanco-García y LindigCisneros, 2005), del que se obtuvieron datos del efecto de factores bióticos y abióticos en el establecimiento de las plantas en los arenales. El experimento inició en julio de 2002 y se evaluó la supervivencia de las plantas de pino 11 y 23 meses después. La tercera etapa consistió en un ex perimento para determinar la supervivencia de plantas de dos especies de pino; Pinus pseudostrobus y $P$. mon tezumae, con dos tratamientos: con y sin presencia de corteza como acolchado. Para la primera especie se uti1 i z a ron plantas de 7-8 meses de edad y de 18 meses, y para la segunda especie plantas de 18 meses. Este experimento inició en julio de 2003 y se cuantificó la supervivencia 11 meses después (Alvarado-Sosa, 2006). Una fuente adicional de información son los ensayos de progenies y procedencias que se han llevado a cabo en la zona (Viveros-Vive ros et al., 2005, 2006), los cuales han permitido identificar algunos de los factores bióticos y abióticos que limitan las transiciones entre estados.

Propuesta de un esquema conceptual para la restau-

\section{ración de bosques de coníferas}

Los bosques de coníferas que constituyen el estado deseable en la zona de trabajo son manejados con fines silvícolas. Estos bosques están dominados por Pinus pseu dostrobus, especie que se encuentra ampliamente distribuida desde una altitud de 1,900 hasta 3,100 m s.n.m., y que se encuentra asociada con P. leiophylla Schiede ex Schltdl. et Cham., P. montezumae Lamb., P. michoacana Martínez (también llamado $P$. devoniana Lindl.) y $P$. douglasiana Martínez, además de varias especies latifoliadas como Crataegus mexicana Moc. et Sessé ex DC., Quercus rugosa Née, Q. laurina Bonpl., Ternstroemia pringlei (Rose) Standl., Alnus jorullensis ssp. lutea Furlow, Oreopanax xalapensis (Kunth) Decne. et Planch. y Salix paradoxa Kunth (Medina et al., 2000). Estos bosques son manejados con el sistema conocido como Método de Desarrollo Silvícola (MDS), el cual consiste en realizar una serie de aclareos que culminan con una corta de regeneración; posteriormente ésta es seguida de una corta de liberación. El efecto de la aplicación del MDS es la modificación de la estructura de los rodales naturales, de una estructura incoetánea a una estructura coetánea, la cual permite un manejo más intensivo.

Como parte de la dinámica de los bosques manejados, encontramos a los claros que se forman por causas naturales o por la corta de árboles como parte del MDS. En estos sitios la regeneración del bosque se da por procesos sucesionales naturales o por la reforestación. También existen terrenos que fueron desmontados con fines agrícolas y que presentan diversos grados de degradación de suelo. En estos terrenos la reforestación, particularmente cuando se encuentran alejados de las fuentes de semillas, es la única estrategia que se ha observado que permite su transición hacia el estado deseable.

El estado perturbado de mayor interés dentro del proyecto de colaboración con la CINSJP hasta la fecha consiste en la vegetación asociada a los depósitos de ceniza volcánica que carecen de una cobertura arbórea y que se conocen localmente como arenales (figura 1). A poco más de 50 años de haber cesado la actividad del volcán Paricutín, los arenales se encuentran casi desprovistos de vegetación. La escasa vegetación presente consiste en manchones dominados por especies arbustivas, separados entre sí por extensiones de arena carentes de cubierta vegetal. Como resultado del esfuerzo de la primera etapa del proyecto, en un área de 1.44 ha ubicadas en el arenal denominado "Mesa de Cutzato", se encontraron 44 manchones de vegetación de diversas dimensiones (de $1.67 \mathrm{~m}^{2}$ a $149.52 \mathrm{~m}^{2}$ ) que en conjunto cubren menos de $15 \%$ del área muestreada. En dichos manchones las especies dominantes, en orden de abundancia, son: Eupatorium glabratum Kunth, Senecio stoechadi formis DC., S. salignus DC. y Muhlenbergia minutissima (Steud.) Swallen. Otras especies comunes, pero no domi- 
nantes, son: Crusea brachyphylla Schltdl. et Cham., C. diversifolia (Kunth) W.R.Anderson, Jaegeria hirta (Lag.) Less., Cyperus seslerioides Kunth y Aegopogon cenchroides Humb. et Bonpl. ex Willd.; Lupinus elegans Kunth es particularmente abundante en islas cercanas a la periferia del arenal. Cuando se compara el incremento en la riqueza de especies que ha ocurrido en los derrames de lava, el cono y los bosques afectados por la erupción del volcán Paricutín, es en los arenales en donde este incremento ha sido más lento (Lindig-Cisneros et al., 2006).

En cinco años en los que se ha visitado de manera sistemática la zona nunca se ha observado el reclutamiento de especies arbóreas en los arenales; de hecho, los pocos ejemplares de árboles que se encuentran en estos sitios son

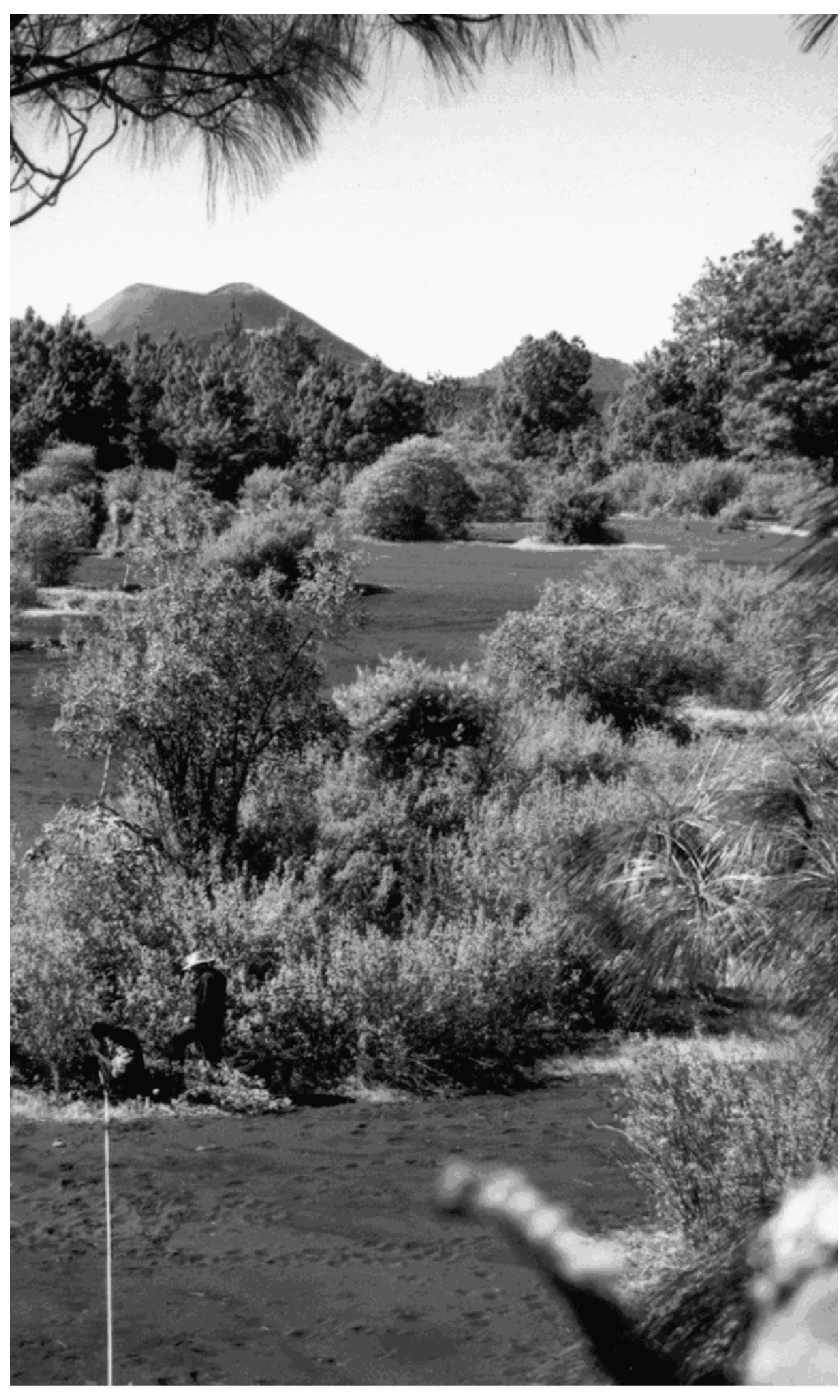

Figura 1. Vista de los manchones de vegetación en el área de estudio en la Mesa de Cutzato, al momento de trazar uno de los transectos de medición y con el cono del volcán Paricutín al fondo. Nótese la arena totalmente desprovista de vegetación entre los manchones y la ausencia de individuos de especies arbóreas. sobrevivientes de plantaciones, principalmente de Pinus pseudostrobus. El desempeño de los árboles en estas plantaciones es pobre, mostrando gran variabilidad en las tasas de crecimiento y supervivencia. En individuos de $P$. pseudostrobus de cuatro años de edad, tanto la distancia a la orilla de la vegetación como la profundidad de la capa de ceniza volcánica limitaron el desempeño de los individuos de P. psedostrobus (Alejandre-Melena, 2004). Los resultados del estudio de los arenales sugieren que éstos constituyen un estado estable caracterizado por una condición de sucesión detenida (cuadro 1).

Con la finalidad de contar con una herramienta de manejo que permita incorporar las necesidades de restauración en la zona, se está elaborando un modelo de estados y transiciones. Con la información generada hasta el momento se proponen tres estados alternativos principales (figura 2) que en orden creciente de disturbio son: (1) bosques manejados para actividades silvícolas (un estado que consiste en un mosaico de estadios sucesionales alterados por la actividad humana), (2) terrenos agrícolas y, (3) arenales (que hasta donde sabemos fueron todos tierras agrícolas al momento de la erupción del volcán Paricutín). En el caso de los terrenos agrícolas, este estado se puede subdivir en

Cuadro 1. Resumen de las características del estado estable presente en los arenales.

\begin{tabular}{|c|c|}
\hline Característica & Descripción \\
\hline Vegetación dominante & $\begin{array}{l}\text { La vegetación está dominada por cuatro es- } \\
\text { pecies características de estados sucesiona- } \\
\text { les tempranos: Eupatorium glabratum, } \\
\text { Senecio stoechadiformis, S. salignus y } \\
\text { Muhlenbergia minutissima. Estas especies } \\
\text { colonizaron unos pocos años después del } \\
\text { término de la erupción (Egler, 1948). }\end{array}$ \\
\hline
\end{tabular}

Carencia de especies Una característica importante es la arbóreas carencia de especies arbóreas, las cuales no han colonizado a pesar de que han transcurrido más de 50 años desde que la erupción terminó.

Barreras que se han identificado para transitar a otros estados

(a) La profundidad de la capa de arena es una barrera importante que limita el desempeño de las plantas, así como la carencia de materia orgánica en este sus trato (Gómez-Romero et al., 2006).

(b) La temperatura que alcanza la arena en la época seca $\left(\mathrm{ca} .60^{\circ} \mathrm{C}, 4 \mathrm{~cm}\right.$ por debajo de la superficie) es una barrera importante que limita la supervivencia de las plantas (Blanco-García y Lindig-Cisneros, 2005). (c) La herbivoría limita el establecimiento de diversas especies, entre las que destaca Lupinus elegans, la leguminosa de mayor talla en la zona (Blanco-García y LindigCisneros, 2005). 


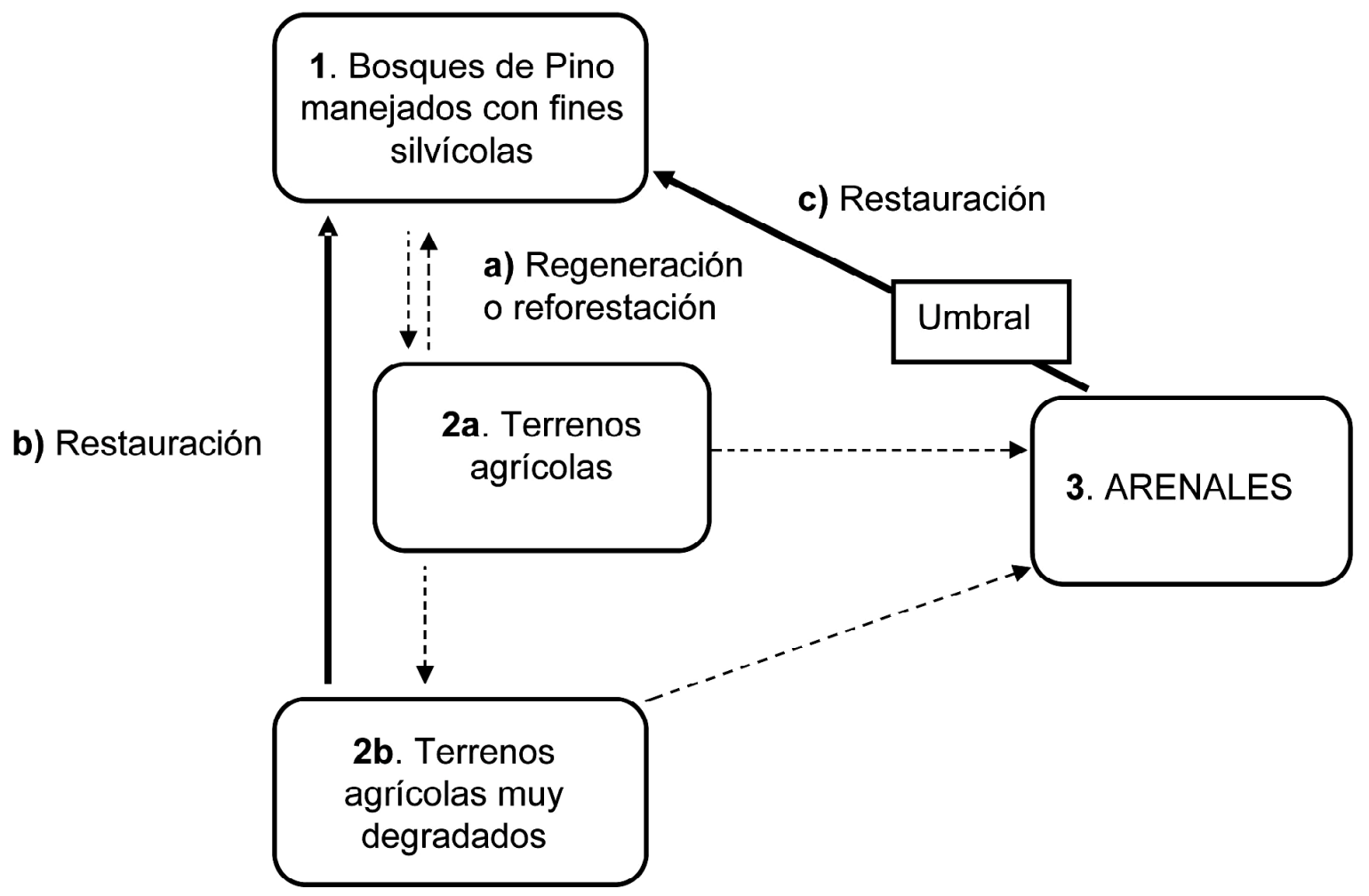

Figura 2. Diagrama de estados y transiciones para la región de estudio. Las líneas punteadas representan cambios debidos al disturbio y las líneas continuas las transiciones causadas por la sucesión secundaria o la restauración. Se propone la existencia de un umbral entre la transición de arenales a bosque maduro.

función de la intensidad, la duración del uso y el tamaño de los terrenos agrícolas, pues todos estos factores pueden crear un gradiente de degradación creciente. Actualmente se llevan a cabo experimentos para evaluar este aspecto del modelo.

Las transiciones que ocurren entre los estados más perturbados a los menos perturbados son las siguientes: (a) regeneración natural de terrenos agrícolas a vegetación secundaria y posteriormente a bosque o regeneración asistida por reforestación, (b) en terrenos agrícolas alejados de las fuentes de diásporas o con niveles altos de disturbio, se puede restaurar el bosque por medio de la reforestación y otras medidas adicionales como la protección de las plantas contra tuzas y otros roedores, o riegos de auxilio (Viveros-Viveros et al., 2005, 2006), y (c) transición de arenales a bosque de pinos, mediante el desarrollo de técnicas de restauración adecuadas para cada caso.

\section{Barreras entre estados}

La dinámica de las perturbaciones naturales es modificada por las perturbaciones antrópicas, creando un mosaico en los ecosistemas que comprende diversos estados, algunos de ellos estables o casi estables. En las tierras de Nuevo San Juan Parangaricutiro, la transición de terrenos agrícolas a bosques se da cuando se abandonan las prácticas agrícolas que mantienen el estado y es auxiliada por la reforestación, particularmente con Pinus pseudostrobus. En la t ra nsición de sitios agrícolas a bosque maduro, de acuerdo con experimentos llevados a cabo hasta la fecha, dos barreras principales limitan el establecimiento de las especies arbóreas, las heladas y la actividad de forrajeo de mamífe ros pequeños, los cuales pueden eliminar a un alto porcentaje de las plantas (hasta un 70\% en el lapso de un año; Viveros-Vive ros et al., 2006). Sin embargo, la regeneración nat ural puede generar la transición entre estos dos estados altemativos.

En el caso de los arenales, la medida de restauración que ha resultado más eficiente para incrementar la supervivencia y el desempeño (evaluado como crecimiento) de Pinus pseudostrobus ha sido el uso de acolchados. El uso de acolchados puede llegar a duplicar la supervivencia de esta especie en años secos. La protección contra el daño mecánico causado por la escorrentía y la mortalidad causada por la herbivoría también permiten que haya una mayor supervivencia de esta especie y de otras, como Lupinus ele gans (Blanco-García y Lindig-Cisneros, 2005). Los diversos experimentos que hemos realizado nos permiten llegar a la conclusión de que éste es el estado que más barreras presenta para transitar hacia bosques. Las pincipales 
barreras que se han identificado hasta el momento para transitar de arenales a bosques incluyen: (1) herbivoría por mamíferos pequeños que pueden eliminar completamente las plantaciones de algunas especies (e.g. conejos han eliminado plantaciones de Lupinus elegans), (2) la profundidad de la capa de arena, que representa una barrera no lineal para el establecimiento de las especies vegetales (Alejandre-Melena, 2004; Gómez-Romero et al., 2006); (3) las bajas concentraciones de nutrientes en la ceniza volcánica, y (4) las altas temperaturas que alcanza la arena en la época seca del año, lo cual aparentemente es la barrera más importante. Estas barreras sugieren que los arenales constituyen un estado estable, pues la composición florística es diferente de la de otros estados, además de que se presentan cambios temporales no lineales causados por eventos catastróficos (Scheffer et al., 2001). Por tal razón, existen múltiples barreras que impiden que el sistema transite al estado que presentaba antes del cambio y se requiere de intervención para llevarlo a otros estados. Es decir, los arenales posiblemente constituyan un estado de sucesión detenida. La sucesión detenida ha sido reportada en bosques afectados por perturbaciones antrópicas (Paul et al., 2004; Ruprecht, 2005) y otros tipos de situaciones en los cuales las condiciones bióticas y abióticas impiden que se transite a estados alternativos (Chapman, 1999; Zanne y Chapman, 2001; Griscom et al., 2003), en particular a estados sucesionales más avanzados.

Las medidas de intervención aplicadas sobre varios factores de manera simultánea, como por ejemplo la reducción de la temperatura del sustrato por medio de acolchados, la reforestación para paliar las limitaciones de la dispersión, el establecimiento de especies arbóreas y el uso de fertilizantes para contrarrestar la carencia de nutrientes y de leguminosas para incorporar nitrógeno y materia orgánica al sustrato, probablemente tengan el potencial de permitir la transición entre el estado de arenal al de bosque.

Con base en el modelo que se está desarrollando, se puede concluir que el efecto combinado del disturbio antrópico que transforma a la vegetación nativa en terrenos agrícolas, y de las perturbaciones naturales consecuencia de los fenómenos volcánicos, crea un estado de perturbación (los arenales) cuya transformación en bosque implica cruzar uno o varios umbrales. Para ello, se requiere realizar acciones específicas de restauración o dejar transcurrir mu cho tiempo para dar cabida a un proceso sucesional muy lento; a su vez, éste podría llevar al sistema a estados no deseables desde el punto de vista del manejo.

\section{Agradecimientos}

Deseamos agradecer a la Dirección Técnica Forestal de la comunidad indígena de Nuevo San Juan Parangaricutiro por el apoyo brindado a lo largo de los años, al Gaylord Nelson Institute for Environmental Studies (GNIES-UW) y al Department of Botany de la Universidad de WisconsinMadison, el apoyo económico de GNIES-UW a través de un donativo del Sr. Ed Weigner, al CONACYT (SEMARNAT2002-CO1-0760) y a la Coordinación de la Investigación Científica de la Universidad Michoacana de San Nicolás de Hidalgo (Proyectos 8.15). Deseamos agradecer los comentarios de Ángela Saldaña Acosta y un revisor anónimo sobre versiones previas de este manuscrito.

\section{Literatura citada}

Alejandre-Melena N. 2004. Estudio sobre el establecimiento de Pinus pseudostrobus Lindl. en sitios afectados por deposición de ceniza volcánica en las inmediaciones del volcán Paricutín, Michoacán, México. Tesis de Licenciatura, Facultad de Biología, Universidad Michoacana de San Nicolás de Hidalgo, Morelia, 152 pp.

Alvarado-Sosa P.A. 2006. Ensayo de especies y de coberturas para restaurar sitios con distinto grado de disturbio. Tesis de Maestría, Facultad de Biología, Universidad Michoacana de San Nicolás de Hidalgo, Morelia, 86 pp.

Allen-Diaz B. y Bartolome J.W. 1998. Sagebrush-grass vegetation dynamics: comparing classical and state-transition models. Ecological Applications 8:795-804.

Blanco-García A. y Lindig-Cisneros R. 2005. Incorporating restoration in sustainable forestry management: using pine bark mulch to improve native-species establishment on tephra deposits. Restoration Ecology 13:703-709.

Bradshaw A.D. 1984. Ecological principles and land reclamation practice. Landscape Planning 11:35-48.

Briske D.D., Fuhlendorf S.D. y Smeins F.E. 2005. State-and-transition models, thresholds, and rangeland health: a synthesis of ecological concepts and perspectives. Rangeland Ecology and Management 58:1-10.

Chapman C.A., Chapman L.J., Kaufman L. y Zanne A.E. 1999. Potential causes of arrested succession in Kibale National Park, Uganda: growth and mortality of seedlings. African Journal of Ecology 37:81-92.

Christensen N.L., Bartuska A.M., Brown J.H., Carpenter S., D’Antonio C., Francis R., Franklin J.F., MacMahon J.A., Noss R.F., Parsons D.J., Paterson C.H., Turner M.G. y Woodmansee R.G. 1996. The report of the Ecological Society of America committee on the scientific basis for ecosystem management. Ecological Applications 6:665-91.

Egler W.A. 1948. Plant communities in the vicinity of the volcano Paricutín, Mexico, after two and a half years of eruption. Ecology 29:415-437.

Ellis J.E. y Swift M. 1988. Stability of African pastoral ecosystems: alternate paradigms and implications for development. Journal of Range Management 41:450-459.

Gómez-Romero M., Lindig-Cisneros R. y Galindo-Vallejo S. 2006. Effect of tephra depth on vegetation development in areas affected by volcanism. Plant Ecology 183:207-213.

Griscom B.W. y Ashton P.M.S. 2003. Bamboo control of forest succession: Guadua sarcocarpa in Southeastern Peru. Forest Ecology and Management 175:445-454.

Hobbs R.J. y Mooney H.A. 1993. Restoration ecology and invasions. En: Saunders D.A., Hobbs R.J. y Ehrlich P.R. Eds. Nature Conservation 3: Reconstruction of Fragmented 
Ecosystems, Global and Regional Perspectives, pp. 127-133, Surrey Beatty and Sons, Sidney.

Hobbs R.J. y Norton D.A. 1996. Towards a conceptual framework for restoration ecology. Restoration Ecology 4:93-110.

Holling S.C. 1973. Resilience and stability of ecological systems. Annual Review of Ecology and Systematics 4:1-23.

Lindig-Cisneros R., Desmond J., Boyer K.E. y Zedler J.B. 2003. Wetland restoration thresholds: Can a degradation transition be reve rsed with increased effort? Ecological Applications 13:193-205.

Lindig-Cisneros R., Galindo-Vallejo S. y Lara-Cabrera S. 2006. Vegetation development in agricultural fields cove red by tephra after 50 years of the eruption of the Paricutín volcano, Mexico. Southwestern Naturalist 51:455-461.

Lindig-Cisneros R. y Zedler J.B. 2000. Restoring urban habitats, a comparative study. Ecological Restoration 18:185-194.

May R.M. 1977. Thresholds and breakpoints in ecosystems with multiplicity of stable states. Nature 269:471-477.

Medina C., Guevara-Féfer F., Martínez M.A., Silva-Sáenz P., Chávez-Carbajal M.A. y García I. 2000. Estudio florístico en el área de la comunidad indígena de Nuevo San Juan Parangaricutiro, Michoacán, México. Acta Botanica Mexicana 52:5-41.

Palmer M.A., Ambrose R.F. y Poff N.L. 1997. Ecological theory and community restoration ecology. Restoration Ecology 5:291-300.

Paul J.R., Randle A.M., Chapman C.A. y Chapman L.J. 2004. Arrested succession in logging gaps: is tree seedling growth and survival limiting? African Journal of Ecology 42:245-251.

Ruprecht E. 2005. Secondary succession in old-fields in the Transylvanian Lowland (Romania). Preslia 77:145-157.

Scheffer M., Carpenter S., Foley J.A., Folke C. y Walker B. 2001. Catastrophic shifts in ecosystems. Nature 413:591-596.

Suding K.N., Gross K.L. y Houseman G.R. 2004. Alternative states and positive feedbacks in restoration ecology. Trends in Ecology and Evolution 19:46-53.

Fecha de recepción: 24 de junio de 2005

Versión corregida: 13 de agosto de 2006

Aceptado: 29 de septiembre de 2006
Tamzen K.S., Krueger C.K. y Thomas D.R. 2001. Application of non-equilibrium ecology to rangeland riparian zones. Journal of Range Management 54:210-217.

Van Nes E.H., Scheffer M., van den Berg M.S. y Coops H. 2002. Dominance of charophytes in eutrophic shallow lakes-when should we expect it to be an alternative stable state? Aquatic Botany 72:275-296.

Viveros-Viveros H., Sáenz-Romero C., López-Upton J. y VargasHe rnández J.J. 2005. Variación genética altitudinal en el crecimiento de plantas Pinus pseudostrobus Lindl. en campo. Agrociencia 39:575-587.

Viveros-Viveros H., Sáenz-Romero C., Vargas-Hernández J.J. y López-Upton J. 2006. Variación entre procedencias de Pinus pseudostrobus establecidas en dos sitios en Michoacán, México. Revista Fitotecnia Mexicana 29:121-126.

Westoby M., Walker B. y Noy-Meir I. 1989. Opportunistic management for rangelands not at equilibrium. Journal of Range Management 42:266-274.

Yates C.J. y Hobbs R.J. 1997. Woodland restoration in the western Australian wheat-belt: a conceptual framework using a state and transition model. Restoration Ecology 5:28-35.

Zanne A.E. y Chapman C.A. 2001. Expediting reforestation in tropical grasslands: distance and isolation from seed sources in plantations. Ecological Applications 11:1610-1621.

Zedler J.B. 1999. The ecological restoration sprectrum. En: Streever W. Ed. An International Perspective on Wetland Rehabilitation, pp. 301-318, Kluwer Academic Publishers, Dordrecht.

Zedler J.B. 2000. Progress in wetland restoration ecology. Trends in Ecology and Evolution 15:402-407.

Zedler J.B. 2003. Wetlands at your service: reducing impacts of agriculture at the watershed scale. Frontiers in Ecology and the Environment 1:65-72.

Zedler J.B. y Callaway J.C. 2000. Evaluating the progress of engineered tidal wetlands. Ecological Engineering 5:211-225. 